\title{
Ocupación romana de la cueva de Los Toriles (Carrizosa, Ciudad REAL): PRIMEROS TESTIMONIOS Y TRASCENDENCIA
}

\author{
Roman frequentation of the Los Toriles cave (Carrizosa, Ciudad Real): first testimonies and implications
}

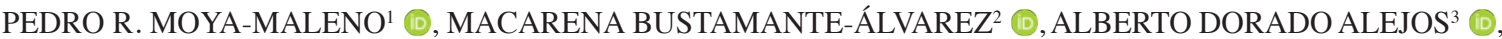

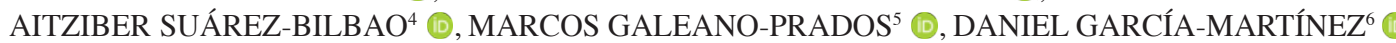

(1) Dpto. Prehistoria, $\mathrm{H}^{\mathrm{a}}$ Antigua y Arqueología. Universidad Complutense de Madrid. Centro de Estudios del Campo de Montiel. preyesmo@ucm.es

(2) Dpto. Prehistoria y Arqueología. Universidad de Granada. UNIARQ-Lisboa.mbustamante@ugr.es

(3) Dpto. Prehistoria y Arqueología. Universidad de Granada.doradoalejos@ugr.es

(4) Dpto. Geología. Universidad del País Vasco. aitziber.suarez@ehu.eus

(5) marcos.ga.pr@gmail.com

(6) Dpto. Biodiversidad, Ecología y Evolución. Universidad Complutense de Madrid. Centro de Estudios del Campo de Montiel. dangar29@ucm.es

\section{RESUMEN:}

Se dan a conocer los primeros testimonios de frecuentación romana de la cueva de Los Toriles (Carrizosa, Ciudad Real), una gruta con una larga ocupación animal y humana, cuanto menos desde el Pleistoceno y hasta nuestros días. Aunque en la primera campaña de excavación de la cueva sólo se han podido constatar algunos fragmentos de cerámica, estos son lo suficientemente relevantes como para confirmar el conocimiento y entrada a la cavidad durante la Antigüedad. Además del análisis tipológico y arqueométrico de las cerámicas, esta novedosa cronología permite reflexionar sobre estos y otros casos similares de época romana, acerca de la infrarrepresentación del fenómeno en la meseta Sur, así como sobre el contexto inmediato del ager laminitanus.

Palabras Clave: Los Toriles, Carrizosa, cueva, terra sigillata, Ager laminitanus.

\section{Abstract:}

This paper presents the first testimonies of Roman frequentation of the Los Toriles cave (Carrizosa, Ciudad Real), a cave with a continued animal and human occupation, at least since the Middle Pleistocene and up to the present day. Although in the first campaign of excavation of the cave only a few ceramic fragments have been found, these are relevant enough to confirm the knowledge and occupation of the cavity during Antiquity. In addition to the typological and archaeological analysis of the ceramics, this unexpected chronology allows us to reflect on these and other similar cases from the Roman period, on the underrepresentation of the phenomenon in the Southern Plateau, as well as on the immediate context of the ager laminitanus.

Key words: Los Toriles, Carrizosa, cave, Terra Sigillata, Ager laminitanus.

Copyright: (C) 2021 Pedro R. Moya-Maleno et al. This is an open access paper distributed under the 
La cueva de Los Toriles se encuentra en el costado oriental del valle de cañada de Pozo Hondo (Carrizosa, Ciudad Real), en una garganta de unos $40 \mathrm{~m}$ de ancho que conduce hasta el cercano río Cañamares (fig. 1).

La cueva que nos centra es una cavidad en "L" con una profundidad sumada de unos $29 \mathrm{~m}$. Su piso se va elevando hasta la zona más profunda explorada, a más de $330 \mathrm{~cm}$ respecto a la superficie del exterior (fig. 2). Se ha definido cuatro subáreas que se han denominado visera, boca, codo o sala, todas con material arqueológico o paleontológico, siendo en las dos últimas donde se localizaron las piezas que tratamos en este trabajo (GarcíaMartínez et al. 2020).

Las primeras lecturas geológicas de la cueva la datan hace más de 70.000 años -fecha mínima por U-Th- sobre un sistema kárstico generado sobre estratos paleolacustres y travertínicos. La primera campaña que se desarrolló en el sitio en 2019 ha confirmado la presencia de comunidades de la Edad del Bronce, pero la cronología se dilata ampliamente antes y después de la Prehistoria Reciente (GarcíaMartínez et al. 2020; Megía-García et al. 2020). En este trabajo se valora la posible frecuentación del sitio en época romana a tenor de unos restos cerámicos que analizamos sucintamente en las páginas venideras.

\section{LOS MATERIALES ROMANOS: CARACTERÍS- TICAS TIPOLÓGICAS Y ARQUEOMÉTRICAS}

Si bien durante la campaña de 2019 se han documentado restos cerámicos a mano y a torno que, con visión amplia de este tipo de producciones y de las comunidades que convivieron durante varios siglos a caballo del cambio de era, podrían adscribirse también a cronologías

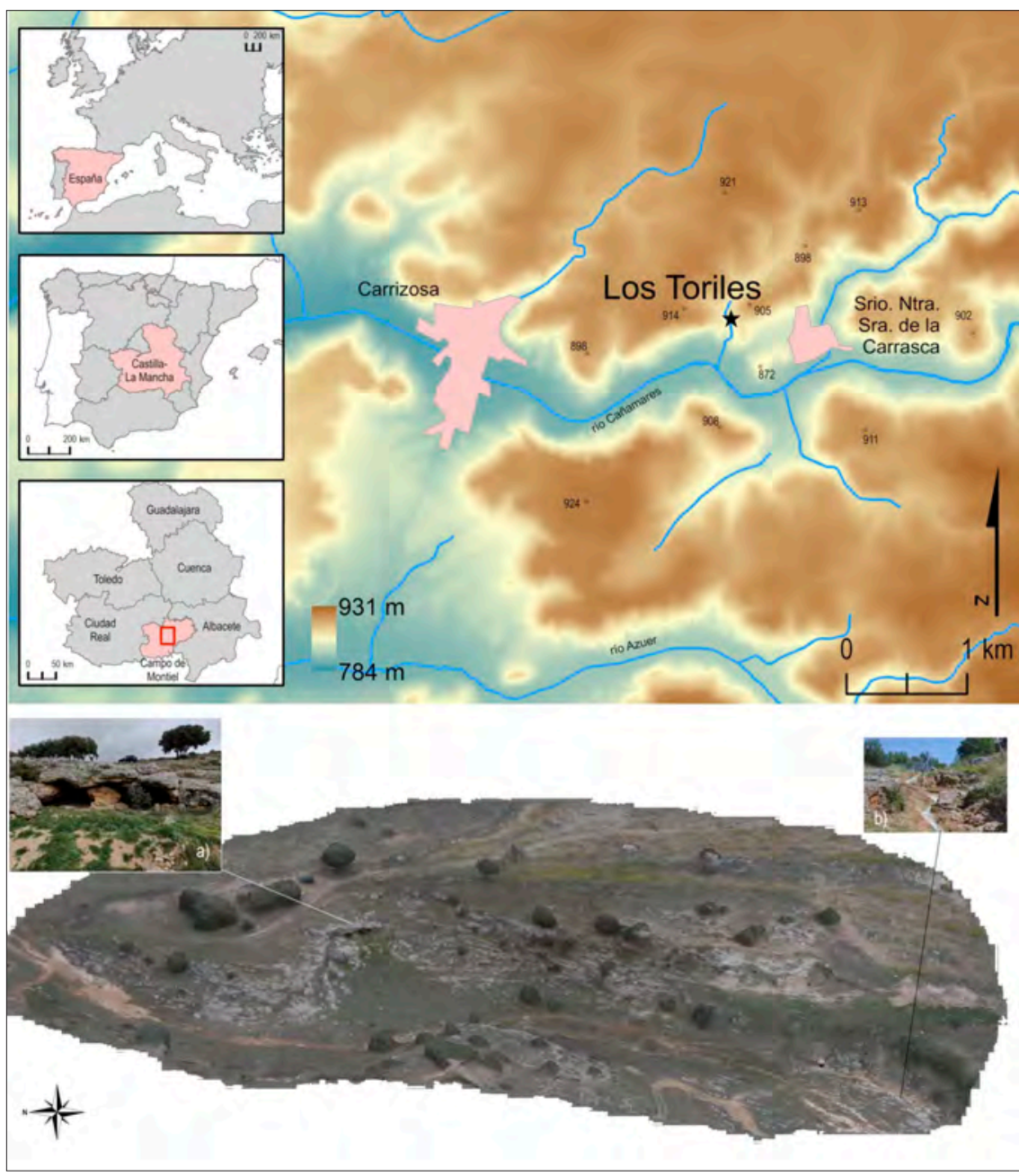

Fig. 1: Mapa de situación de la cueva de Los Toriles (arriba) y recreación fotogramétrica de la Cañada de Pozo Hondo (abajo) con detalle de la entrada (a) y del arroyo (b). 


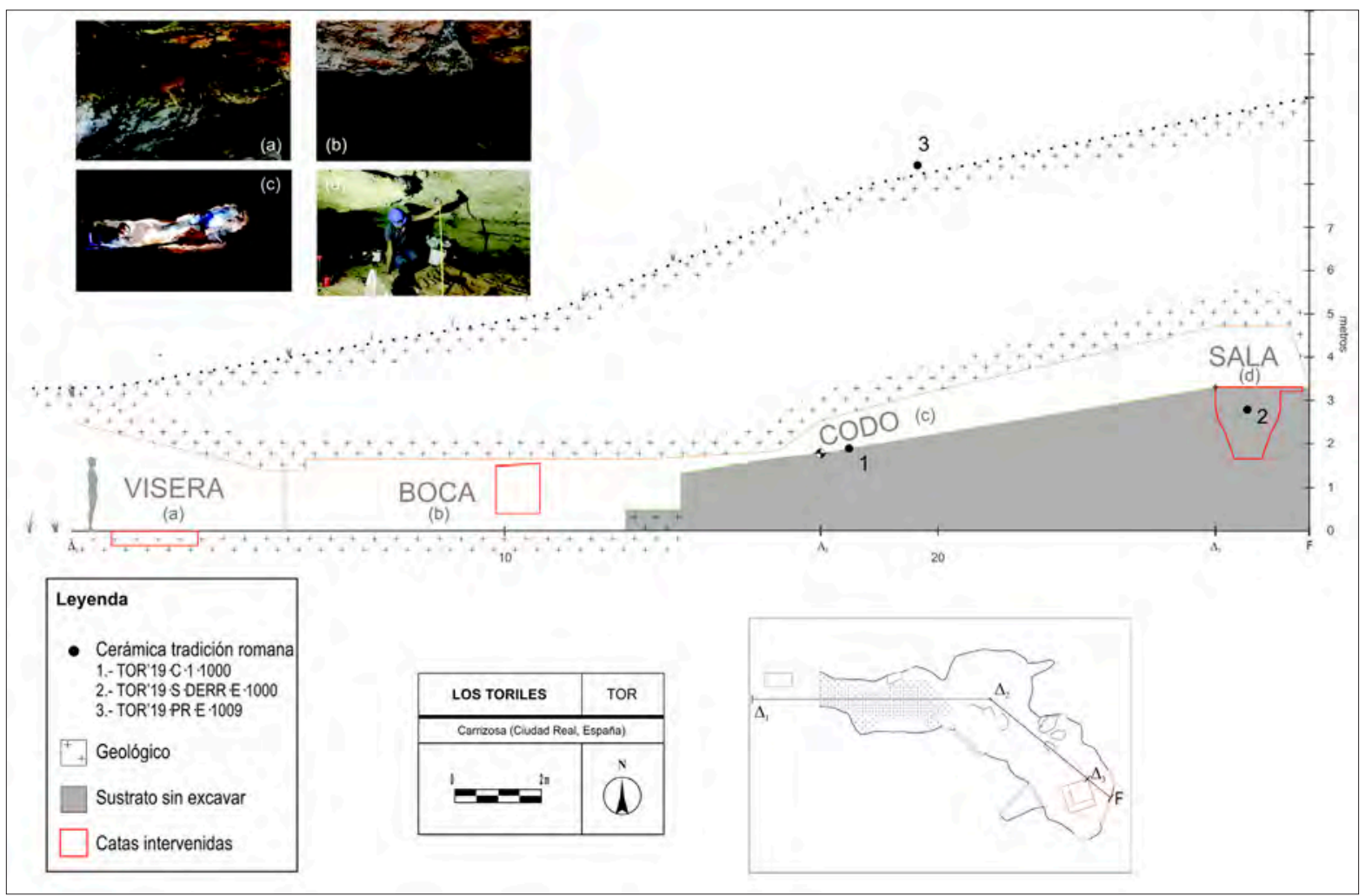

Fig. 2: Sección y planta aproximadas de la cueva de Los Toriles, con áreas de intervención y de localización de las cerámicas estudiadas en el artículo (con indicación con punto negro $\mathrm{n}^{\circ} 1-3$ ).

hispanorromanas, lo fragmentado del registro y la prudencia invitan a centrarnos en aquellos materiales cuya factura es inequívocamente de tradición romana. Sirvan estas evidencias para plantear nuevas hipótesis de partida de una cueva de la que, hasta el momento, se conocía únicamente su ocupación prehistórica.

Estos son tres fragmentos, dos en terra sigillata procedentes del interior de la cueva (TOR'19.C $\cdot 1 \cdot 1000$ y TOR'19·S·DERR·E·1000) y uno de cerámica de imitación tipo Peñaflor localizado en los aledaños de la cavidad (TOR'19·PR·E·1009; 2) (fig. 3). Su presencia en un contexto de difícil acceso como éste apunta directamente a la frecuentación del sitio y no a hallazgos casuales ni a procesos postdeposicionales.

\section{TOR' $19 \cdot C \cdot 1 \cdot 1000$}

Este fragmento de terra sigillata fue localizado en el codo de la cueva debajo de un gran bloque caído de la techumbre (fig. 2). Es un fragmento de fondo plano de $c a .13 \mathrm{~cm}$ de diámetro y pie elevado con leve indicación al exterior. La pasta es de tonalidad marronácea oscura (Cailleux R-17) muy depurada, amasada y contiene algún nódulo calcáreo de granulometría media (fig. 4, d; fig. 5, a). Presenta un recubrimiento sinterizado marrón rojizo (Cailleux R-17) (fig. 3, 2) brillante fruto de una elevada cocción que raya la vitrificación favoreciendo una adherencia perfecta con la arcilla (fig. 4, a-c). Cuenta con amplio espesor de recubrimiento que, incluso, generó que el alfarero imprimiera sus huellas dactilares en el anillo del pie al sacar la pieza del recipiente que contuvo dicho recubrimiento líquido (fig. 4, f). Queda para el futuro analizar en detalle este paleodermatoglifo.

El análisis macroscópico de la pieza revela una producción cuidada, realizada en ambientes oxidantes. La matriz, de tono pardo, presenta una importante cantidad de pequeñas inclusiones $(\mathrm{ca} .5 \%$ ) inferiores a $1 \mathrm{~mm} \mathrm{e}$ identificadas como posibles fragmentos de $\mathrm{CaO}$, junto a pequeñas reducciones de tonos oscuros que podrían relacionarse con pequeños contenidos de materia orgánica (fig. 5, a). La orientación de estas inclusiones es oblicua debido al modelado de la vasija mediante torno. Por su 


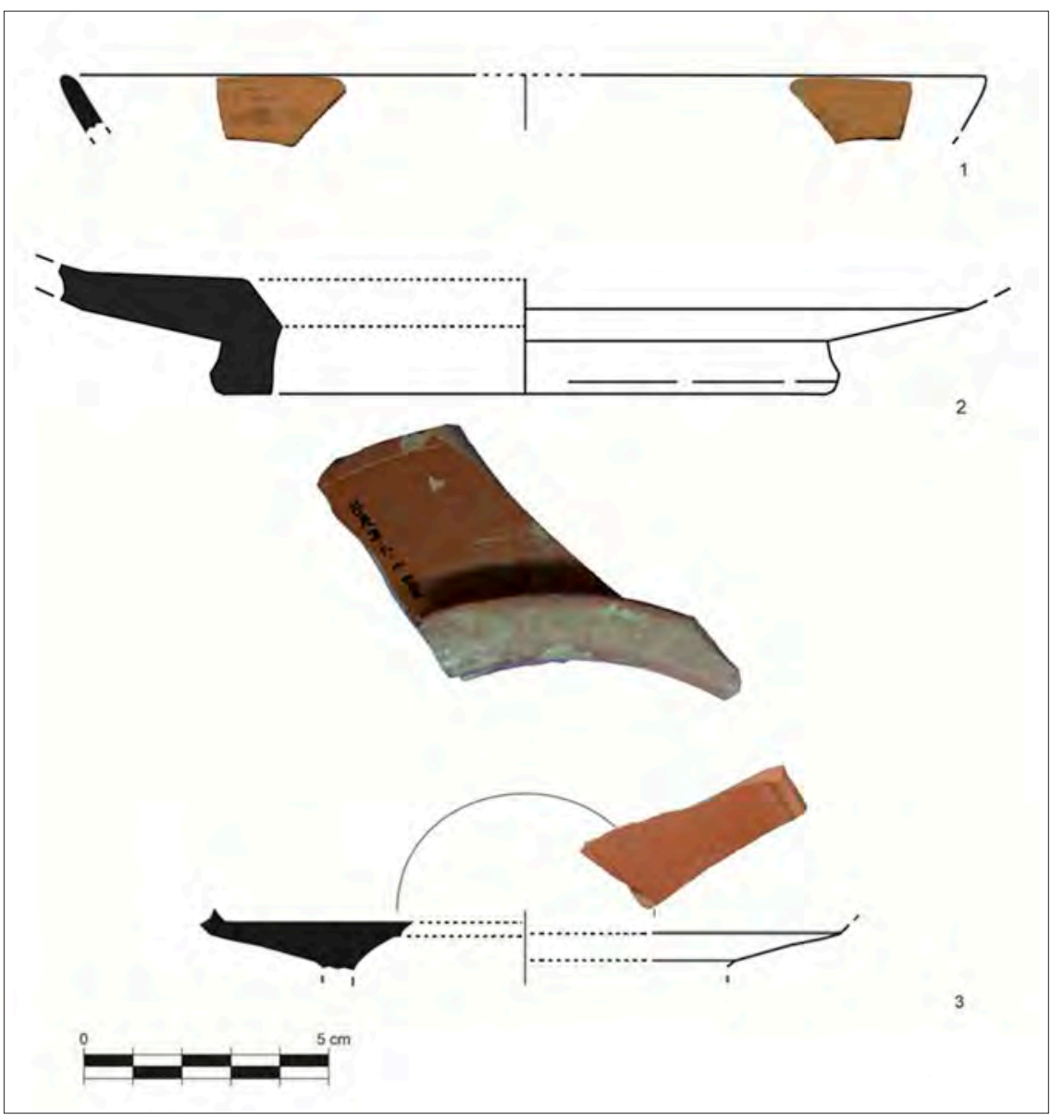

Fig. 3: Lámina de formas de los fragmentos cerámicos de tradición romana documentados en la primera campaña de la cueva de Los Toriles: 1) TOR'19.PR E' 1009; 2) TOR'19. C.1·1000; y 3) TOR' $19 \cdot S \cdot D E R R \cdot E \cdot$ 1000.

parte, los pocos poros que aparecen aquí son de muy pequeño tamaño y están relacionados con el proceso de secado y cocción, localizados preferentemente en la zona central de la matriz. El engobe documentado en sendas superficies presenta una alta calidad y buen grado de sinterización. Sin embargo, pueden observarse pequeñas vacuolas cuyo origen podemos vincular a las temperaturas alcanzadas durante el proceso de cocción en el horno (fig. 5, b).

Interpretativamente, estamos ante un gran plato de pie elevado de clara conformación augustea vinculado tipológicamente a las conocidas páteras del servicio 1 de Haltern (Consp. 10-11). Sin embargo, a pesar de la adopción morfológica de una forma itálica, la arcilla posee importantes nódulos cálcicos algo que se aleja de la realidad arqueométrica de estas producciones. Ante esto, proponemos como hipótesis de trabajo que el lugar de origen de esta singular pieza fueran los talleres sucursales itálicos en Lyon. Sin embargo, esta propuesta necesitará ser confirmada a partir de análisis comparativos arqueométricos con piezas procedentes de este taller. Recordemos que, hasta el momento, uno de los más conocidos, el de la Muette, ha sido definido como un taller galo que manufactura al italico modo confectae. Este taller surge como respuesta de la demanda que la cerámica itálica estaba teniendo en este arco mediterráneo que supuso no sólo la transferencia de conocimientos técnicos a esta zona sino también un posible trasvase de figuli así como de instrumental o incluso arcillas (Picon y Garmier 1974; Picon y Lasfargues 1974; Genin et al. 2002; Desbat 2000). La presencia de esta cerámica en la península Ibérica está atestiguada por los sellos que evidencian su origen (Beltrán 1990: 67). Además, se cimienta en la aparición de otras producciones de la zona más fáciles de adscribir macroscópicamente como son las paredes finas ampliamente difundidas en el arco NO peninsular (Morillo y García Marcos 2003: 298-300). 
Fig. 4: Fotografías generales y macroscópicas de la cerámica TOR'19. C. 1000 .

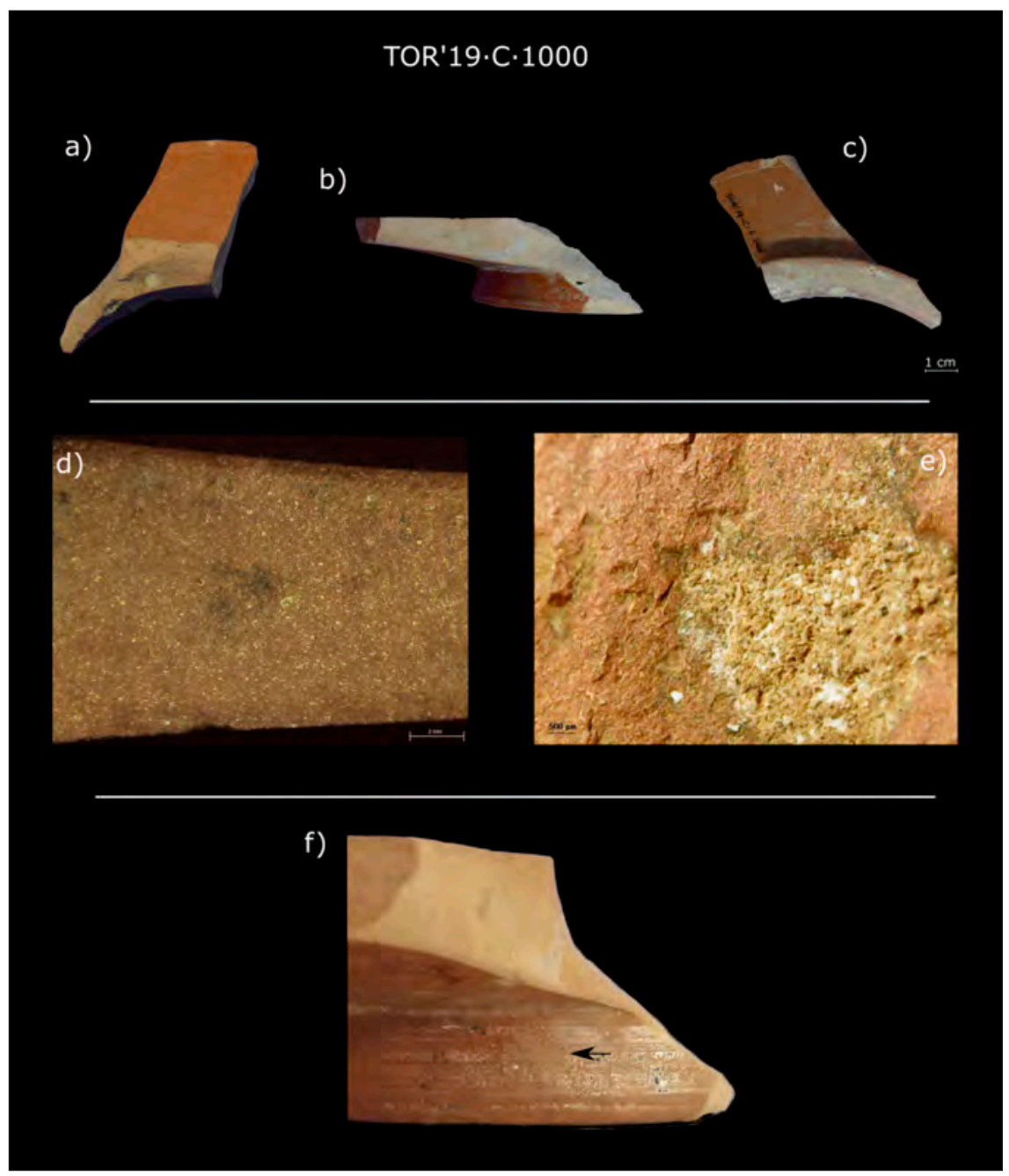

En el entorno del enclave que estamos analizando se ha atestiguado como paralelo, una posible variante de las Consp. 10-11 en el enclave hispanorromano de Libisosa, Lezuza (Poveda y Sánchez 2018: 279).

Ante esto podemos concluir que estamos ante una gran pátera itálica de producción provincial cuya manufactura se debió focalizar en el cambio de era. En relación a su función, claramente, estamos ante un recipiente destinado al servicio e ingesta de alimentos.

\section{TOR'19·S·DERR·E· 1000}

Este fragmento apareció en la cata de la "Sala", en el lugar más profundo de la cueva al que hoy día tenemos acceso (fig. 2). Apareció dentro de un paquete que se derrumbó del perfil E de la cata durante la excavación.
Aunque no se sabe exactamente de qué nivel artificial procede, no cabe duda de su presencia entre los niveles 0-V (Prehistoria Reciente-Edad Contemporánea).

La cerámica es un fragmento de la carena entre el cuerpo y la base de un plato. En la parte superior se observa la presencia de una incisión central que marcaría un círculo de unos ca. $51 \mathrm{~mm}$ que podría haber delimitado el sigillum. Presenta recubrimiento marrón anaranjado mate (Cailleux R-40) y craquelado exterior fruto de una fuerte temperatura de cocción (fig. 6, e). Su pasta está muy depurada, con apenas nódulos calcáreos (fig. 5, c; fig. 6, e). Su coloración es marronácea clara (Cailleux N-47). La circunferencia que traza la carena permite definir un diámetro de base que alcanza los $c a .12,3 \mathrm{~cm}$. El fondo es claramente plano hasta que llega a la carena, la cual se presenta exvasada (fig. 3, 3). 


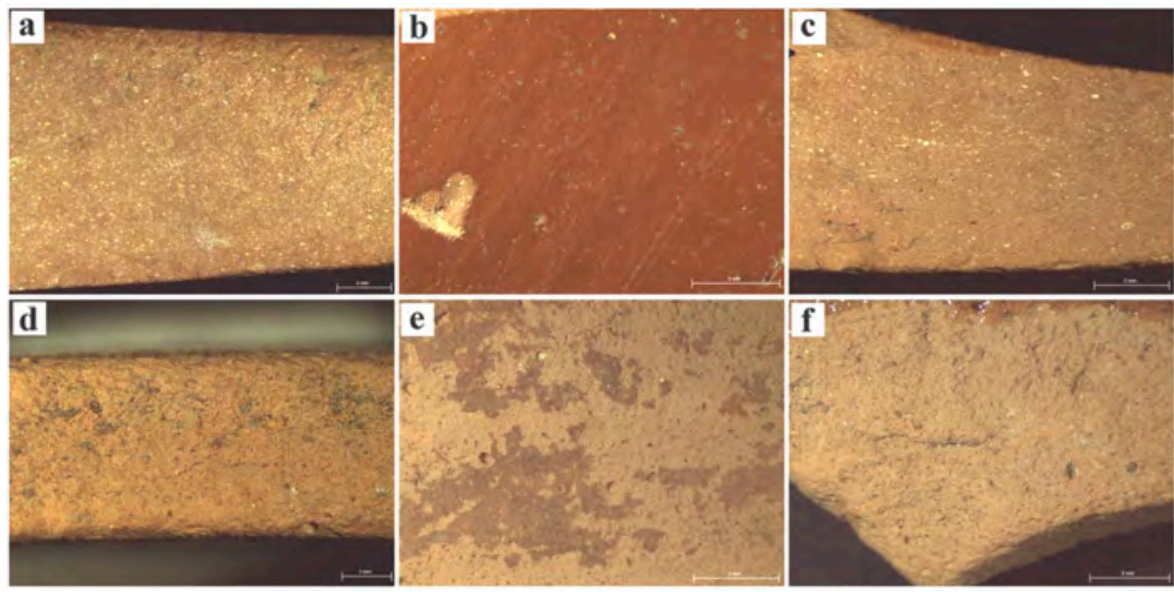

\section{TOR'19·S·DERR·E·1000}

a)

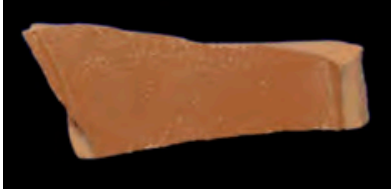

b)

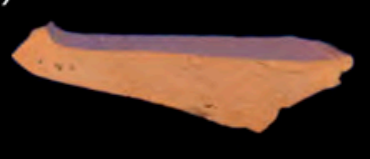

c)

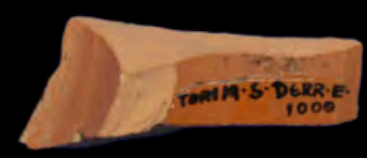

$1 \mathrm{~cm}$ d)

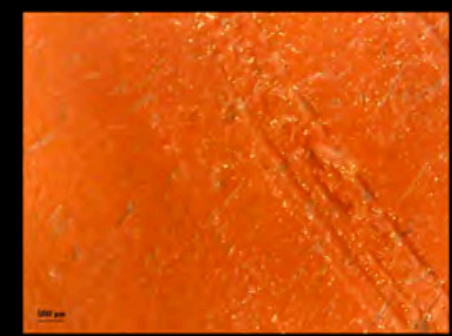

Fig. 5: Fotografías mediante lupa estereoscópica de las matrices y engobes de TOR' $19 \cdot \mathrm{C} \cdot 1 \cdot 1000(\mathrm{a}-\mathrm{b}), \mathrm{TOR}^{\prime} 19 \cdot \mathrm{S}$. DERR·E'1000 (c) y TOR'19. PR.E'1009 (d-f).

Fig. 6: Fotografías generales y macroscópicas de la cerámica TOR'19·S·DERR·E·1000.

\section{f)}

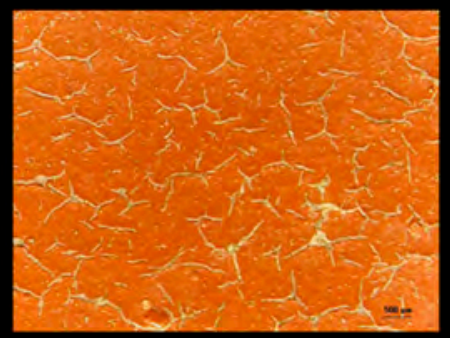


Fig. 7: Fotografías generales y macroscópicas de la cerámica TOR'19 PR-E 1000 .

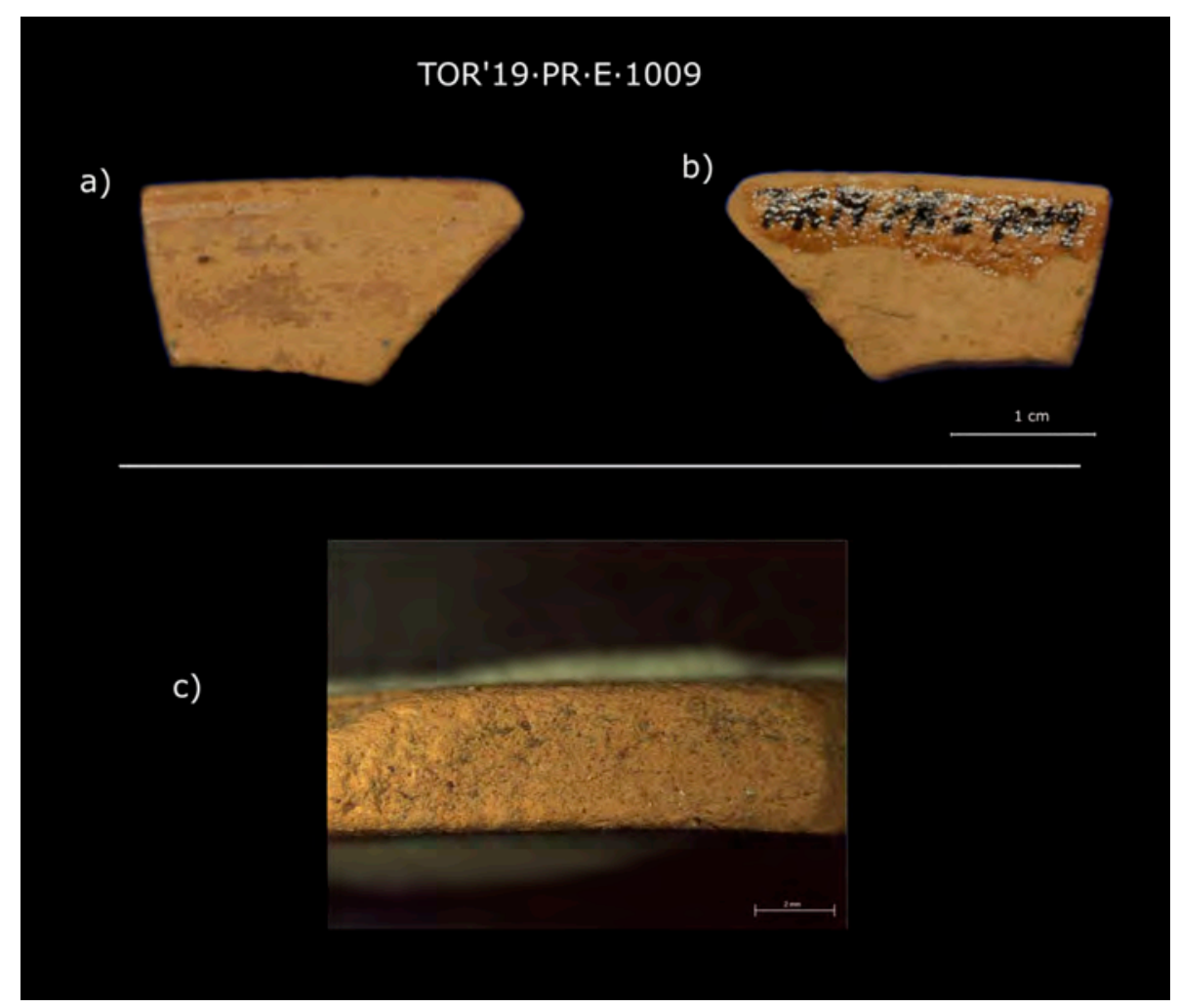

Macroscópicamente es una cerámica muy similar a la anterior. Estamos ante una producción cuidada, realizada en ambientes oxidantes. La matriz es parda con tintes ligeramente más claros- presenta menores cantidades de inclusiones $(<2 \%)$, inferiores a $0,1 \mathrm{~mm}$ e igualmente identificadas como posibles fragmentos de $\mathrm{CaO}$ (fig. 5, c). Igualmente, la orientación de estas inclusiones es oblicua debido al modelado de la vasija mediante torno. Se observan algunas estrías en las zonas externas, también relacionadas con el proceso de secado y cocción, localizados preferentemente en la zona central de la matriz. El engobe documentado presenta una alta calidad con escasas vacuolas que, como en el caso anterior, son fruto de la fase de secado y cocción.

A pesar del grado de obliteración podemos concluir que se trata de un plato de producción gálica, concretamente de los talleres de La Graufesenque. La dificultad que presenta la ausencia de elementos diagnósticos -como bordes o fondos-obstaculizan su posible adscripción cronológica, aunque no descartamos que formara parte de un plato del tipo Drag. 18, una forma ya constatada en otros puntos de la provincia (Fuentes 2015: 143 ss).
TOR'19·PR·E·1009

Pequeño fragmento de labio aparecido en superficie por encima de la cueva durante una inspección del terreno. Presenta un recubrimiento externo con doble acabado, al interior brillante y adherente (Cailleux P-20) y al exterior parcialmente perdido (fig. 7, a-b). La pasta es granulosa y de coloración rosácea (Cailleux M-20) (fig. 5, d; fig. 7, c). Tampoco mantiene perímetro suficiente como para conocer su diámetro con exactitud, si bien rondará los 10-15 cm (fig. 3, 1).

Este vaso difiere de las producciones anteriores en el análisis macroscópico. Cocido en ambientes oxidantes, la matriz ofrece unas tonalidades ligeramente más anaranjadas en la que se documentan un mayor número de desgrasantes $(5-7 \%)$, identificados como cuarzos $(<0,3$ $\mathrm{mm}$ ) de morfología redondeada, y algunas micas y nódulos de $\mathrm{CaO}$ de forma accesoria heterogéneamente distribuidas en la matriz (fig. 5, d). La orientación preferencial de estos desgrasantes tiende a ser horizontal, que podría vincularse a producciones realizadas a molde lo que, por otro lado, permite señalar también las estrías formadas a lo largo de la pared y de forma paralela a esta. El engobe aplicado en este caso es de mala calidad, lo que ha provocado que se 
pierda casi totalmente. Este problema podría tener relación con las menores temperaturas alcanzadas en el horno, lo que habría provocado que tal película no se fijara adecuadamente (fig. 5, e-f).

A pesar de sus reducidas dimensiones, concluimos que estamos ante una cerámica de imitación tipo Peñaflor de la forma del tipo Mart. Ib. Tradicionalmente se ha establecido su dispersión en la zona S peninsular pero los nuevos hallazgos perfilan una distribución más allá del espacio meridional. Para la región castellano-manchega los hallazgos, aunque limitados, permiten hablar de un comercio puntual en estos momentos. Al respecto, los procedentes de las Minas de Diógenes (Solana del Pino) (Domergue 1967: 76; Martínez 1989), Sisapo (Zarzalejos 1995) o Lezuza (Poveda 1994) son claros ejemplos de este fenómeno. La procedencia de este fragmento podría apuntar a los talleres de la Bética suroccidental por su carácter pulverulento, bien del área de Celti (Keay y Romo 2001) o bien de la bahía de Cádiz (Bustamante y López Rosendo 2014). En relación a la cronología, los análisis desarrollados en Augusta Emerita focalizan este tipo de formas a fines del periodo julioclaudio (Bustamante-Álvarez 2013: 69).

\section{RESULTADOS GEOQUÍMICOS}

La caracterización geoquímica de las tres muestras mediante un espectrómetro PANalytical modelo Zetium de Fluorescencia de Rayos X en la Universidad de Granada ha permitido la identificación de los elementos mayoritarios $\mathrm{SiO}_{2}, \mathrm{Al}_{2} \mathrm{O}_{3}, \mathrm{CaO}, \mathrm{Fe}_{2} \mathrm{O}_{3}, \mathrm{MnO}, \mathrm{Na}_{2} \mathrm{O}, \mathrm{K}_{2} \mathrm{O}$, $\mathrm{TiO}_{2}, \mathrm{P}_{2} \mathrm{O}_{5}$ y los minoritarios $\mathrm{Zr}, \mathrm{Sr}, \mathrm{Cr}, \mathrm{Ba}, \mathrm{Rb}, \mathrm{Zn}$ y V (fig. 8). Al análisis estadístico mediante Análisis de Componentes Principales (ACP) (Aitchison 1983) se han añadido 47 análisis más con el fin de ampliar el espectro comparativo (Compana et al. 2010; 2013; 2014; Madrid y Buxeda 2013; Serrano 2016: 360, tab. 7.3, 449 ss; Serrano et al. 2016). Así, aunque no se corresponde con el mismo tipo de producción, atendemos al principio de procedencia (Weigand et al. 1977; Cau 2003: 155; Tsantini 2007: 132).

Los resultados muestran que las producciones identificadas como TSG y TSI de producción provincial no poseen una relación geoquímica con ninguno de los principales centros productores peninsulares conocidos hasta la fecha lo que, por otro lado, no sorprende dada su tipología; esto nos permite, por otro lado, ver la coherencia de los resultados estadísticos (figs. 8 y 9). De igual modo, la producción tipo Peñaflor tampoco parece relacionarse con las analizadas por Madrid y Buxeda (2013), indicativo de su dispar procedencia. Sin embargo, existe un importante vacío analítico en relación a estas producciones que deberá ser suplido en trabajos futuros. A tenor de la gran cantidad de piezas de este tipo documentadas en suelo peninsular, con seguridad debieron existir más talleres alfareros. Este incremento del muestreo y su consecuente análisis permitirá en un futuro generar datos más robustos sobre sus posibles centros de producción.

\section{CONTEXTO E INTERPRETACIÓN}

Aunque la reconstrucción paleoambiental de la garganta muestra un bosque mediterráneo abierto antropizado durante los últimos dos milenios, la prospección

\begin{tabular}{|c|c|c|c|}
\hline Sample & TOR'19・PR・E・1009 & TOR'19・S・DERR・E・1000 & TOR'19・C・1・1000 \\
\hline $\mathrm{SiO}_{2}$ & 61,38 & 53,37 & 51,44 \\
\hline $\mathrm{Al}_{2} \mathrm{O}_{3}$ & 20,94 & 22,16 & 22,46 \\
\hline $\mathrm{CaO}$ & 1,95 & 9,79 & 12,01 \\
\hline $\mathrm{Fe}_{2} \mathrm{O}_{3}$ & 7,03 & 5,94 & 5,65 \\
\hline $\mathrm{MnO}_{\mathrm{Na}_{2} \mathrm{O}}$ & 0,03 & 0,05 & 0,05 \\
\hline $\mathrm{K}_{2} \mathrm{O}$ & 0,16 & 0,23 & 0,29 \\
\hline $\mathrm{TiO}_{2}$ & 2,51 & 3,87 & 3,78 \\
\hline $\mathrm{P}_{2} \mathrm{O}_{5}$ & 11,100 & 10,600 & 10,300 \\
\hline $\mathrm{Zr}$ & 0,1000 & 0,3700 & 0,3500 \\
\hline $\mathrm{Sr}$ & 0,0252 & 0,1635 & 0,1499 \\
\hline $\mathrm{Cr}$ & 0,0099 & 0,0293 & 0,0392 \\
\hline $\mathrm{Ba}$ & 0,0144 & 0,0140 & 0,0126 \\
\hline $\mathrm{Rb}$ & 0,0346 & 0,0276 & 0,0272 \\
\hline $\mathrm{Zn}$ & 0,0050 & 0,0166 & 0,0166 \\
\hline $\mathrm{V}$ & 0,0039 & 0,0129 & 0,0112 \\
\hline & 0,0168 & 0,0690 & 0,0080 \\
\hline
\end{tabular}

Fig. 8: Datos geoquímicos obtenidos para las muestras analizadas procedentes de Ciudad Real (expresados en \%). 
Fig. 9: Resultado del ACP obtenido a partir de los datos geoquímicos de las muestras analizadas procedentes de Ciudad Real junto con otros datos disponibles para otros centros productores peninsulares.

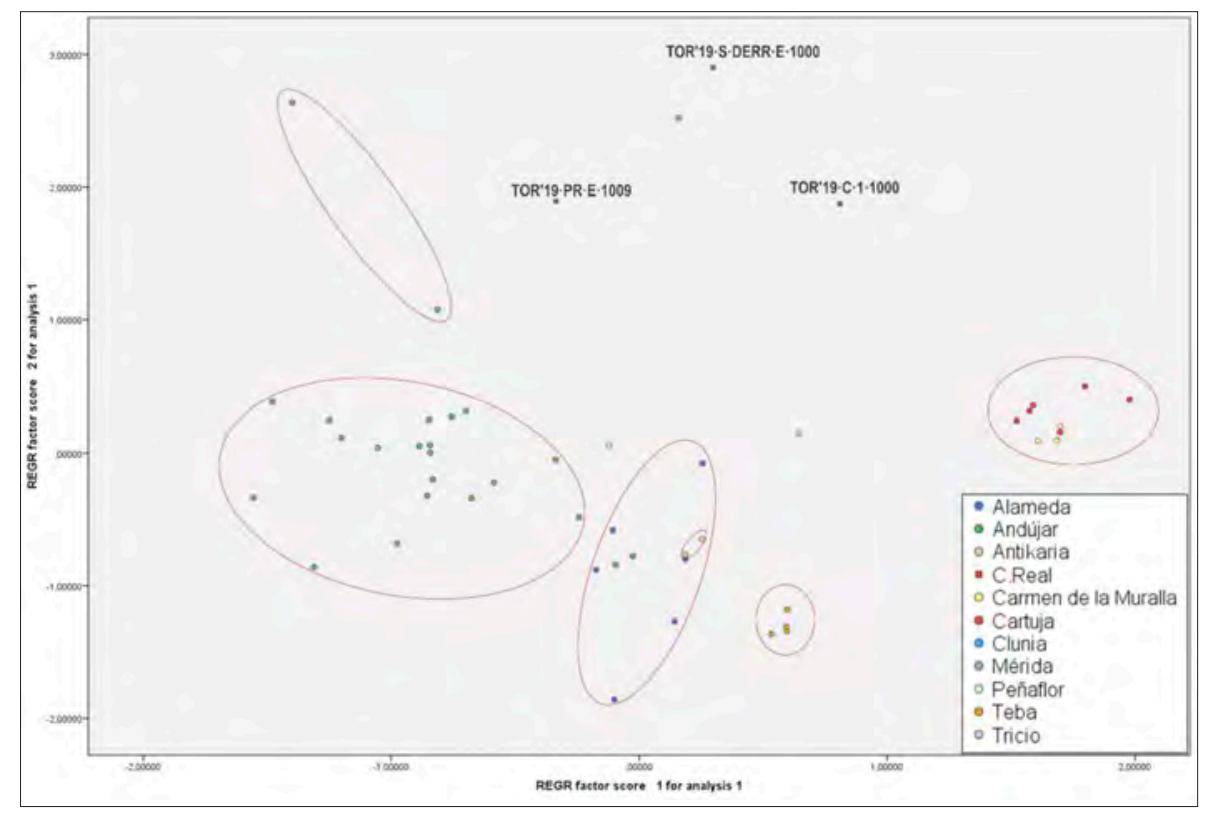

intensiva de la parcela ${ }^{1}$ no muestra materiales romanos más allá del fragmento tipo Peñaflor localizado en el entorno de la gruta. De no haber ningún hábitat inmediato, estaríamos ante un polo de atracción y frecuentación de asentamientos más lejanos.

La cueva se encuentra en una zona bastante abrupta de la comarca, separada considerablemente de los principales lugares y vías de comunicación romanas: las rutas de primer orden distan entre 5-8 km (Rodríguez Castillo 2008; Roldán y Caballero 2014: 161-173) y los testimonios tipo villae más próximos -sin excavar-a unos $4 \mathrm{~km}$. Cabe sospechar de la necrópolis ibérica de Carrizosa, a unos 2,3 km (Jiménez y Chaparro 1981), cuyo poblado pudo acabar romanizado a tenor de una inscripción funeraria del s. I d. C. allí localizada (Alföldy 1987: 248 §19), aunque ésta bien pudiera proceder de cualquier punto de la órbita laminitana como en otros casos (Moya y Novillo 2009: 189 ss) (fig. 10).

Igualmente destacable es otra cueva con materiales romanos en la comarca, la de Montesinos (Ossa de Montiel, Albacete), a más de 17 km en línea recta al NE. De esta famosa cavidad proceden restos alto y bajoimperiales en superficie junto a la boca y a su laguna interior (Jiménez 1996: 5 ss, 17-23, láms. B-F; Jiménez y Chaparro 1989: 91).

Sea como fuere, parece evidente que Los Toriles, $a b$ silentio de otros indicadores como la terra sigillata hispánica, fue frecuentada en el primer siglo de nuestra era y bajo la órbita territorial y económica de Laminium
(Alhambra), un importante cruce de caminos entre la Alta Andalucía y la Meseta a $12 \mathrm{~km}$ y uno de los municipios más romanizados de la Oretania (Domingo 2001; Moya-Maleno 2008; Carrasco y Fuentes 2020).

En cuanto a la función de la cueva durante época romana, estamos ante contextos difíciles de sistematizar por la amplia cronología, calidad y/o ambigüedad del registro que necesitará completarse con futuros hallazgos (inter alia Apellaniz et al 1967; San Nicolás 1985; Bernal et al. 2008; Gutiérrez et al. 2012: 239243; Tobalina et al. 2015). Puesto que a día de hoy en Los Toriles carecemos de inhumaciones, distintos equipamientos cerámicos, tesorillos o testimonios de explotación, hemos de descartar el uso funerario, la exploración, de cantera o un hábitat pastoril o rupestre (sensu Gutiérrez et al. 2012: 243). El relativamente escaso valor de la TS en comparación con otros elementos suntuarios también descarta una intencionalidad de ocultación. Es por ello que consideramos más viable el uso económico, para resguardar un pequeño rebaño o fresquera; o el uso mágico-religioso, como lugar de contacto con algún numen o divinidad de las profundidades, el ultramundo -o incluso de las aguas que todavía hoy se dice oír-, a las que se le realizarían ofrendas con fines propiciatorios, ya fueran cívicos o individuales (inter alia Lorrio et al. 2006; Alfayé 2016; Panosa et al. 2014). En uno y otro caso, lo importante sería el contenido de productos naturales o procesados allí depositados. 


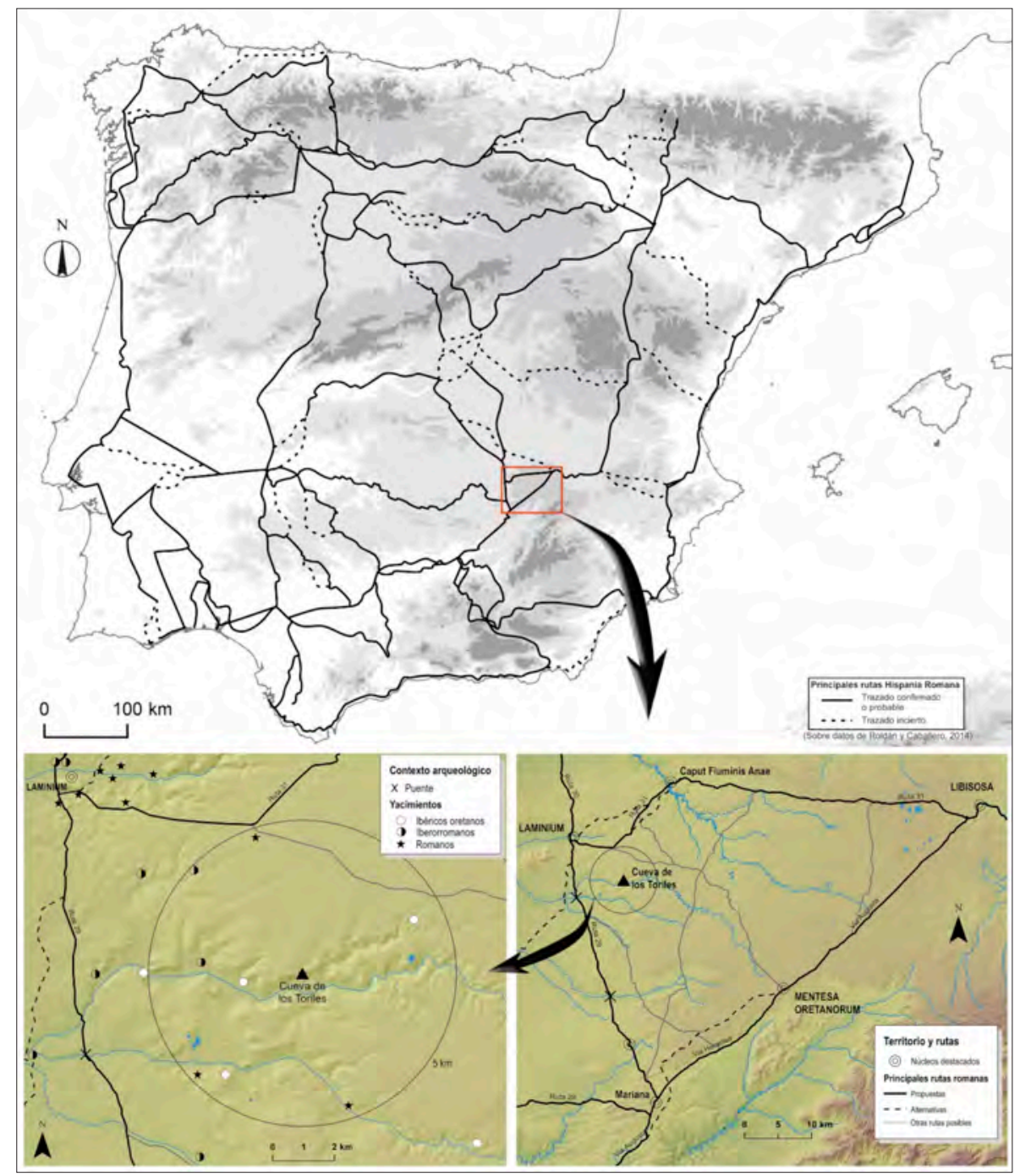

Fig. 10: Vías de comunicación principales en la comarca y yacimientos con materiales oretanos y romanos en el área de la cueva de Los Toriles.

Con todo, en este primer siglo d.C. estamos ante una sociedad mixta y donde la cultura material romana no puede ser indicador inequívoco de la romanidad de su poseedor, sino que en esta cueva, al igual que en el resto del territorio, pudieron estar operando gentes locales con tradiciones prerromanas pero con una progresiva "romanización material” (Fuentes 1992: 590 ss; Woolf 1998). Así se constata, por ejemplo, en alguna urna cineraria ibérica con pátera de TS en la necrópolis del camino del Matadero de Alhambra (Madrigal y Fernández 2001: 239 y fig. 4.3). Este contexto de transición concuerda aquí si entendemos las cerámicas de imitación tipo Peñaflor como un fenómeno de idiosincrasia y reticencia indígena a incorporar las producciones típicamente romanas (Vaquerizo et al. 2005: 182; Bustamante-Álvarez 2013: 70 ss) durante una implantación romana de lenta génesis. De hecho, la cronología julio-claudia de nuestras sigillatas nos sitúan en el contexto la reformulación del territorio en torno a antiguos núcleos oretanos, como Laminium, así como a asentamientos rurales romanos ex novo de la zona. Véase a este respecto cómo cuevas-santuario como la de Gorham (Gibraltar) languidecen con la romanización y la erección de nuevos espacios de culto en zonas urbanas cercanas, caso de Carteia (Belén y Pérez 2000: 534; Bernal et al. 2008: 171). No obstante, la inexistencia hasta la fecha de algún fósil director de tradición oretana o de otros elementos votivos indígenas (Cazorla y Celestino 2008) nos impiden caracterizar quién o quiénes pudieron realiza la deposición de estas piezas y su por qué en la cueva. 


\section{CONCLUSIONES}

Las piezas presentadas, aunque poco numerosas dado el estadio inicial de la investigación, suponen una muestra inequívoca de la frecuentación en época romana de este lugar. Esto se reafirma por el estado de conservación de las piezas y por su contexto en altura y de difícil acceso, lo que anula la llegada de estas piezas por procesos postdeposicionales.

La localización prácticamente intacta en Los Toriles de dos fragmentos de platos de TS destinados al servicio e ingestión de productos sólidos o semisólidos -y no a su preparación- y su ubicación en las partes más profundas de cavidad, así como otro tipo Peñaflor al exterior de la misma, apunta hacia un fenómeno de frecuentación de cuevas, cuando menos, en la primera mitad del I d.C. en esta parte de la submeseta $\mathrm{S}$, en una fase transicional de las poblaciones autóctonas hacia un consumo de piezas plenamente romanas.

Estos testimonios, a expensa de nuevas excavaciones y hallazgos más fecundos, nos exhortan a estudiar su uso en relación con otros casos hispanos similares así como a contextualizar cómo estuvo operando esta cavidad con núcleos menores o principales del ager laminitano y dentro del comercio entre la Gallia y el centro y $\mathrm{S}$ peninsular. Finalmente, pone de manifiesto la infrarrepresentación de este proceso de ocupación de cuevas en la región, no sólo por los condicionantes geomorfológicos, sino también por la carencia de investigaciones al respecto como se evidencia también en la de Montesinos.

\section{NOTA}

1. Prospección realizada a finales de $2020, \mathrm{~N}^{\circ}$ Exp. JCCM: 202057-P1.

\section{AGRADECIMIENTOS}

Agradecemos la colaboración del propietario de la finca, del Ayuntamiento de Carrizosa y de cuantos trabajaron en la cueva o nos han brindado apoyo bibliográfico (M. Almagro-Gorbea, J.C. Guisado y J.A. Hierro). También a los mecenas vía Experiment.com y la Sociedad Española de Paleontología.

Este trabajo se enmarca en el proyecto Corpus Vasorum Hispanorum. Analisis tipológico, cronológico y prosopográfico de los sigilla en terra sigillata hispánica a partir de los centros consumidores. Parte I: Lusitania (PGC2018-093478-A-I00 - Convocatoria Proyectos de Excelencia - Plan Estatal de Generación de Conocimiento -
Ministerio de Ciencia, Innovación y Universidades - España). A. Suárez-Bilbao es receptora de un contrato de doctores recientes hasta su integración en programas de formación postdoctoral en la UPV/ EHU (DOKBERRI), código: DOCREC20/01

\section{BIBLIOGRAFÍA}

AITCHISON, J. (1983): Principal Component Analysis of Compositional Data, Biometrika 70 (1), 57-65. DOI: https://doi.org/10.1093/biomet/70.1.57

ALFAYÉ VILLA, S. (2016): Expresiones religiosas en las ciudades del poder de la Hispania Céltica: el caso de Clunia, Revista de Historiografía 25, 355-383.

DOI: https://doi.org/10.20318/revhisto.2017.3596

ALFÖLDY, G. (1987): Epigraphica Hispanica IX. Inschriften aus Ciudad Real, Zeitschrift für Papyrologie und Epigraphik 67, 225-248.

APELLANIZ, J. M.; NOLTE, E; ALTUNA, J. (1967): Cuevas sepulcrales de Vizcaya. Excavación, estudio y datación por el C 14, Munibe, XIX (3/4), 159-226.

BELÉN, M.; PÉREZ, I. (2000): Gorham's Cave, un santuario en el Estrecho. Avance del estudio de los materiales cerámicos, ACFP 4, II, 531-542.

BELTRÁN LLORIS, M. (1990): Guía de la cerámica romana, Pórtico, Zaragoza.

BERNAL, D.; DOMÍNGUEZ, J. C.; RAISSOUNI, B. (2008): Las cuevas en el Círculo del Estrecho en época histórica. Una línea de investigación arqueológica con futuro, Las ocupaciones humanas de la cueva de Caf Taht el Ghar (Tetuán): los productos arqueológicos en el contexto del estrecho de Gibraltar (J.R. Muñoz, M. Zouak, D. Bernal Casasola, B. Raissouni coords), Cádiz, 153-189.

BUSTAMANTE-ÁLVAREZ, M. (2013): La terra sigillata hispánica en Augusta Emerita. Estudio tipo cronológico a partir de los vertederos del suburbio norte, Anejos de AEspA LXV, CSIC, Mérida.

BUSTAMANTE-ÁLVAREZ, M.; LÓPEZ ROSENDO, E. (2014): Las imitaciones locales de terra sigillata de la Bahía de Cádiz, Comer a la moda. Imitaciones de vajilla de mesa en Turdetania y la Bética occidental durante la Antigüedad (VI a.C.-Vi d. C.) (F. J. García Fernández, E. García Vargas), Universitat de Barcelona, 271-300.

CARRASCO SERRANO, G.; FUENTES SÁNCHEZ, J. L. (2020): Comercio de terra sigillata hispánica altoimperial del ámbito de tritium magallum en la provincia de Ciudad Real, Economía romana en Castilla-La Mancha (G. Carrasco, coord), Cuenca, 389-435.

CAU ONTÍVEROS, M. A. (2003): Cerámica tardorromana de cocina en las Islas Baleares: Estudio arqueométrico, B.A.R.I.S. 1182, Oxford. DOI: https://doi.org/10.30861/9781841715490 
Pedro M. Moya-Maleno, Macarena Bustamante-Álvarez, Alberto Dorado Alejos, Aitziber Suárez-Bilbao, Marcos Galeano-Prados, Daniel García-Martínez

CAZORLA MARTíN, R.; CELESTINO PÉREZ, S. (2008): La Cueva del Valle (Zalamea de La Serena, Badajoz): un santuario rupestre en la comarca de La Serena, SPAL 17, 207-232.

DOI: https://doi.org/10.12795/spal.2008.i17.09

COMPANA, J. M.; CABEZA, A.; ARANDA, M. A. G.; LEÓNREINA, L. (2014): The Baetican workshops: a starting point to study terra sigillata hispanica, JAS 45, 26-35. DOI: https://doi.org/10.1016/j.jas.2014.01.003

COMPANA, J. M.; LEÓN-REINA, L.; ARANDA, M. A. G. (2010): Archaeometric characterization of terra sigillata hispanica from Granada workshops, Boletín de la Sociedad Española de Cerámica y Vidrio 49, 113-119.

COMPANA, J. M.; LEÓN-REINA, L.; MACÍAS, J.; SERRANO, E.; ARANDA, M. A. G. (2013): El alfar romano de sigillata hispánica de Singilia Barba: una perspectiva arqueométrica, Actas del I Congreso Internacional Sobre Estudios Cerámicos. Homenaje a la Dra. Mercedes Vegas (L. Girón, M. Lazarich, M. C. Lopes, eds.), Cádiz, 627-652.

DESBAT, A. (2000): Les ateliers Lyonnais et Viennois à l'Epoque d'Auguste et leur rapport avec les ateliers padans, Produzione cerámica in área padara tra il II secolo a.C.e il VII secolo d. C.: nuovi dati e prospettive di ricerca (G. P. Brogiolo, G. Olcese, coords.), Mantua, 70-92.

DOMERGUE, C. (1967): La mine antique de Diogenes (Province de Ciudad Real), Mélanges de la Casa de Veĺázquez. DOI: https://doi.org/10.3406/casa.1967.957

DOMINGO PUERTAS, L. A. (2001): La ciudad iberorromana de Laminium: evolución y municipalización, Historia Antiqua XXV, 151-170.

FUENTES DOMÍNGUEZ, A. (1992): Las necrópolis ibéricas y su transformación ante la romanización, Las necrópolis (J. Blánquez, V. Antona del Val, coords.), Madrid, 587-606.

FUENTES SÁNCHEZ, J. L. (2015): El comercio de cerámicas romanas en la provincia de Ciudad Real: las sigillatas altoimperiales, Actas del I Congreso Nacional Ciudad Real y su provincia (M. S. Campos, A. R. del Valle, F. Alía, J. Anaya, eds.), Ciudad Real, 135-169.

GARCÍA-MARTÍNEZ, D. (2019): ¿Dónde están los fósiles manchegos? El sesgo en el registro Paleontológico del Pleistoceno de La Mancha, Aportaciones a la investigación, gestión y difusión del patrimonio del Campo de Montiel (E. Navarro, F. J. Moya, C. Moya, M. A, Serrano, P. Moya-Maleno, eds.), Almedina: Centro de Estudios del Campo de Montiel, 17-43. DOI: https://doi.org/10.30823/recm.02019116

GARCÍA-MARTÍNEZ, D.; VALENCIANO, A.; SUÁREZ BILBAO, A.; PALANCAR MARÍN, C. A.; MEGÍA GARCÍA, I.; MORENO GARCÍA, D.; CAMPAÑA, I.; MOYAMALENO, P. R. (2020): New remains of a primitive badger from Cueva de los Toriles (Carrizosa, Castilla-La Mancha, Iberian Peninsula) suggest a new quaternary locality in the southern Iberian plateau, Journal of Iberian Geology. DOI: https://doi.org/10.1007/s41513-020-00127-y
GENIN, M. ; HOFFMANN, B. ; VERNHET, A. (2002): Les productions anciennes de la Graufesenque, Céramiques de la Graufesenque et autres productions d'époque romaine: nouvelles recherches: hommages à Bettina Hoffmann, Archéologie et Histoire Romaine (M. Genin, A. Vernhet coords.), Montagnac, 45-104.

GUTIÉRREZ CUENCA, E.; HIERRO, J. A.; RÍOS, J.; GÁRATE, D.; GÓMEZ OLIVENCIA, A.; ARCEREDILLO ALONSO, D. (2012): El uso de la cueva de Arlanpe (Bizkaia) en época tardorromana, AEspA 85, 229-251. DOI: https://doi.org/10.3989/aespa.085.012.013

JIMÉNEZ RAMÍREZ, S.; CHAPARRO SABINA, A. (1981): Hallazgo arqueológico en Carrizosa, Lanza 26-3-1981, 10.

JIMÉNEZ RAMÍREZ, S.; CHAPARRO SABINA, A. (1989): Las lagunas de Ruidera en el tiempo, Villanueva de los Infantes.

JIMÉNEZ RAMÍREZ, S. (1996): Guía de la cueva de Montesinos, ermita de San Pedro de Sahelices y castillo de Rochafrida: Parque Natural Lagunas de Ruidera, Iniestares, Madrid.

KEAY, S.; ROMO, A. (2001): Las cerámicas, La arqueología de unas excavaciones (S. Keay, J. Grighton, eds.), Celti (Peñaflor), 1987-1992.

LORRIO, A. J.; MONEO, T.; MOYA, F.; PERNAS, S.; SÁNCHEZ DE PRADO, M. D. (2006): La cueva Santa del Cabriel (Mira, Cuenca): Lugar de culto antiguo y ermita cristiana, Complutum 17, 45-80.

MADRID I FERNANDEZ, M.; BUXEDA I GARRIGOS, J. (2013) Anexo VI: estudio de caracterización arqueométrica de cerámicas finas altoimperiales procedentes de la ciudad romana de Augusta Emerita, Terra Sigillata Hispánica en Augusta Emerita (Mérida, Badajoz). Valoración tipocronológica a partir de los vertederos del suburbio Norte (M. Bustamante, coord.), Mérida, 180-210.

MADRIGAL, A.; FERNÁNDEZ RODRÍGUEZ, M. (2001): La necrópolis ibérica del Camino del Matadero (Alhambra, Ciudad Real), Arqueología funeraria: las necrópolis de incineración (R. García Huertas, J. Morales Hervás coords.), Cuenca, 225-257.

MARTÍNEZ RODRÍGUEZ, M. (1989): Las cerámicas beticas de imitación tipo Peñaflor, BAEAA 26, 60-65.

MEGÍA GARCÍA, I.; PAULOS-BRAVO, R.; CIFUENTES-ALCOBENDAS, G.; PALANCAR, C. A.; SUÁREZ-BILBAO, A.; MOYA-MALENO, P. R.; GARCÍA-MARTÍNEZ, D. (2020): Typo-technological analysis of a bifacial stone tool from Cueva de los Toriles (Carrizosa, CastillaLa Mancha, Iberian Península) and its importance as a new Acheulean site from the Southern Iberian Plateau, Proceedings of the European Association of Human Evolution (ESHE), 77.

MORILLO, A; GARCÍA MARCOS, V. (2003): Importaciones itálicas en los campamentos romanos del norte de Hispania durante el periodo augusteo y julioclaudio, Rei Cretariae Romanae Fautores 38, 295-304. 
MOYA-MALENO, P. R. (2008): Ager y afiladeras. Dos hitos en el estudio del municipio laminitano (Alhambra, Ciudad Real), El territorio de las ciudades romanas (J. Mangas, M. A. Novillo, coords.), Madrid, 557-588.

MOYA-MALENO, P. R.; NOVILLO LÓPEZ, M. A. (2009): El pasado prerromano y romano del Campo de Montiel, Por tierras del Campo de Montiel: Segundo paseo literario (L. Matamoros, J. F. Cuenca coords.), Madrid, 168-191.

PANOSA, M. I.; RODÁ, I.; UNTERMANN, J. (2014): Les inscripcions ibèriques i llantines del Cogull (Les Garrigues, Lleida), $R A P$ 24, 27-40.

PICON, M.; GARMIER, J. (1974): Un atelier d'Ateius à Lyon, Revue Archéologique de l'Est 25 (1), 71-76.

PICON, M.; LASFARGUES, J. (1974). Transfert de moules entre les ateliers d'Arezzo et ceux de Lyon, Revue Archéologique de l'Est 25 (1), 60-69.

POVEDA NAVARRO, A. M. (1994): Nuevos datos sobre aspectos del comercio exportador de la bética hacia el sudoeste de la Tarraconense, Actas del II Congreso de Historia de Andalucía, Córdoba, 391-392.

POVEDA NAVARRO, A. M.; FUENTES SÁNCHEZ, J. L. (2018): Un ejemplo de contextualización cerámica altoimperial: la evidencia del Área II de la colonia romana de Libisosa Foroagustana (Lezuza, Albacete), Estudios para la configuración de las facies cerámicas altoimperiales en el Sur de la Península Ibérica (P. Ruiz Montes, M. V. Peinado, M. I Fernández García, coords.), Albacete, 271-283. DOI: https://doi.org/10.2307/j.ctv170x3t6.17

RODRÍGUEZ CASTILLO, J. (2008): El camino de Alandumbar y el 'Quijote"”, Con los pies en la tierra. Don Quijote en su marco geográfico e histórico: XII Coloquio Internacional de la Asociación de Cervantistas (XII-CIAC) (F. B. Pedraza, R. González, coords.), Argamasilla de Alba, 121-132.

ROLDÁN HERVÁS, J. M.; CABALLERO CASADO, C. (2014): Itinera Hispana: estudio de las vías romanas en Hispania a partir del Itinerario Antonino, el Anónimo de Rávena y los Vasos de Vicarello, Madrid.
SAN NICOLAS DEL TORO, M. (1985): Aportación al estudio de las cuevas naturales de ocupación romana en Murcia, Conventus Carthaginensis a la Chora de Tudmir, Antigüedad y Cristianismo II, 303-309.

SERRANO ARNÁEZ, B. (2016): Estructuras humanas y económicas de producción de la terra sigillata hispánica. Los productores de moldes, Tesis Doctoral, Universidad de Granada.

SERRANO ARNÁEZ, B.; COMPANA, J. M.; FERNÁNDEZGARCÍA, M. I (2016): Chemical and mineralogical characterization of Roman Sigillata moulds from Andújar (Jaén, Spain), JAS: Reports 7, 60-70.

DOI: https://doi.org/10.1016/j.jasrep.2016.03.044

TOBALINA PULIDO, L.; DUMÉNIL, V.; CAMPO, A. (2015): Fréquentations des grottes durant l'époque romaine: le cas de la Navarre, Cuadernos de Arqueología de la Universidad de Navarra 23, 123-161. DOI: https://doi.org/10.15581/012.23.123-161

TSANTINI, E. (2007): Estudi de la producció $i$ la distribució d'àmfores ibèriques en el NE peninsular a travès de la seva caracterització arqueomètrica, Tesis doctoral, Universitat de Barcelona.

VAQUERIZO GIL, D.; GARRIGUET MATA, J. A.;VARGAS CANTOS, S. (2005): La Constancia. Una contribución al conocimiento de la topografía y los usos funerarios en la Colonia Patricia de los siglos iniciales del Imperio, Córdoba.

WEIGAND, P. C.; HARBOTTLE, G.; SAYRE, E. V. (1977): Turquoise source and source analysis: Mesoamerica and the Southwestern U.S.A, Exchange Systems in Prehistory, 15-34. DOI: https://doi.org/10.1016/B978-0-12-227650-7.50008-0

WOOLF, G. (1998): Becoming Roman. The Origins of Provincial Civilization in Gaul, Cambridge. DOI: https://doi.org/10.1017/CBO9780511518614

ZARZALEJOS, M. (1995): Arqueología de la región sisaponense, aproximación a la evolución histórica del extremo $S W$ de la provincia de Ciudad Real (fines del VIII a.C. al II d.C.), Tesis Doctoral, Universidad Autónoma de Madrid. 